\title{
LA CULTURA DE GÉNERO DEL ALUMNADO DE FORMACIÓN PROFESIONAL EN EL ÁMBITO LABORAL
}

\author{
María E. Urrea-Solano \\ mayra.urrea@ua.es \\ Aitana Fernández-Sogorb \\ María del Pilar Aparicio-Flores \\ María J. Hernández Amorós \\ Departamento Didáctica General y Didácticas Específicas \\ San Vicente del Raspeig (Alicante)
}

Fecha de Recepción: 1 Abril 2018

Fecha de Admisión: 10 Abril 2018

\section{RESUMEN}

El problema de la desigualdad de género radica, en gran medida, en la pervivencia de los patrones tradicionales de género que la sociedad asigna a mujeres y a hombres respectivamente. Por ello, el presente estudio se planteó con el propósito de reconocer las creencias estereotipadas que el alumnado de Formación Profesional presenta ante la igualdad de género en la esfera laboral, verificando, además, la existencia de posibles diferencias significativas según el sexo y el nivel de ciclo formativo cursado (grado medio o grado superior). Para el logro de este objetivo se contó con la participación voluntaria de 135 estudiantes de un centro integrado público de Formación Profesional de la provincia de Alicante, quienes cumplimentaron la Escala de Actitudes de Rol de Género. Los resultados obtenidos evidenciaron que el conjunto de participantes presentaba un posicionamiento ambiguo respecto a la igualdad laboral entre mujeres y hombres, sobre todo en lo concerniente a la división sexual del trabajo. La aplicación de la prueba $t$ de Student, por su parte, reflejó la existencia de diferencias estadísticamente significativas en función del nivel de ciclo formativo, pero especialmente del sexo. En este sentido, fueron las alumnas quienes rechazaron más enérgicamente la exclusión de las mujeres de los cargos de responsabilidad y la segregación ocupacional. En cuanto al nivel de ciclo formativo cursado, el alumnado de grado superior fue quien mostró actitudes más proclives hacia la presencia femenina en los espacios de poder y la intervención del padre en la educación familiar. A la vista de tales resultados, se estima necesario diseñar e implementar actuaciones dirigidas a la deconstrucción de las creencias sexistas que el alumnado de esta etapa parece mostrar en el contexto laboral.

Palabras clave: igualdad de género; Formación Profesional; estereotipos de género; sexismo; ámbito laboral 


\title{
LA CULTURA DE GÉNERO DEL ALUMNADO DE FORMACIÓN PROFESIONAL EN EL ÁMBITO LABORAL
}

\begin{abstract}
Gender culture in the workplace among students on vocational training.

The problem of gender inequality is to a large extent due to the survival of the traditional gender roles that society allocates to women and men respectively. The aim of the present study is therefore to identify the stereotypical beliefs presented by students on vocational training as regards gender equality in the workplace and check for the existence of possible significant differences depending on sex and course level studied (intermediate or advanced). To this end, the study relied on the voluntary participation of 135 students from an integrated public vocational training centre in the province of Alicante, all of whom completed the Gender-Role Attitudes Scale. The results showed that the participants as a whole presented an ambiguous stance as far as equality in the workplace is concerned, especially as regards the sexual division of labour. The Student's t-test indicated that there were statistically significant differences depending on the level of training studied and, in particular, gender. Regarding the latter, it was the female students who most emphatically rejected the exclusion of women from positions of responsibility and occupational segregation. As for the level of training, students at advanced level showed attitudes more favourable to the presence of women in positions of power and a father's involvement in bringing up children. Given these results, we believe there is a need to plan and implement actions aimed at deconstructing the sexist beliefs that students at this stage appear to show in the workplace.
\end{abstract}

Keywords: gender equality; vocational training; gender stereotypes; sexism; workplace

\section{ANTECEDENTES}

El principio de igualdad entre mujeres y hombres y la no discriminación se halla, por definición, en la esencia de los derechos universales del ser humano, constituyendo, además, una de las principales exigencias para que una sociedad pueda definirse a sí misma como democrática y plural. Pero a pesar de ello, y de su reconocimiento en numerosos tratados internacionales, las mujeres se siguen viendo obligadas a enfrentar la desigualdad en escenarios que se suponen igualitarios y equitativos (Burton, 2015; Gagliarducci y Paserman, 2012; Govender y Penn-Kekana, 2008; Morrison, Bourke y Kelley, 2005). Uno de estos entornos lo constituye, sin lugar a dudas, el terreno profesional, donde si bien la participación de la mujer se ha ido incrementando de manera notable, todavía hoy en día se siguen apreciando múltiples desequilibrios. En el contexto estadounidense, por ejemplo, durante el año 2015 las mujeres ganaron un 19\% menos de sueldo medio que sus contrapartes masculinas en el mismo puesto de trabajo (U. S. Bureau of Labor Stastistics, 2017). Porcentajes todavía más elevados se alcanzan en Europa. De acuerdo con el 2017 Report on equality between women and men in the EU (European Union, 2017), incluso entre los estados con políticas de igualdad más activas, la brecha salarial en el año 2016 se situó en torno al 20\%, llegando a alcanzar el $45 \%$ en Alemania 0, inclusive, tasas más elevadas en países como Malta (46\%) o los Países Bajos $(48 \%)$. A la brecha salarial, se ha añadir además la temporalidad y el pluriempleo que también suelen ser más frecuentes entre la población femenina (European Union, 2017; INE, 2017; U.S. Bureau of Labor Statistics, 2017).

Su presencia en los espacios de poder y decisión tampoco se ve libre de escollos y limitaciones, sobre todo en las posiciones más elevadas y de mayor responsabilidad. En el caso de Europa, por ejemplo, solo representan el $23.9 \%$ de los consejos de administración de las compañías que cotizan en bolsa y solo Francia (42.1\%), Suecia (36.9\%), Italia (32.3\%) y Finlandia (30.1\%) se acercan tímidamente a la composición paritaria (European Union, 2017). En el ámbito estadounidense, por su parte, solo el $6.4 \%$ de las compañías Fortune-500 está presidido por una mujer (Catalyst, 2017), Ilegando incluso a cifras más bajas en Europa, donde únicamente lideran el $5.7 \%$ de las empresas CEO (European Union, 2017). Estas irregularidades en la dirección también se advierten 
en el escenario español. Según el informe Women in Business 2017 (Grant Thornton, 2017), centrado en el liderazgo femenino mundial, aunque nuestro país se sitúe por encima de la media europea, el índice de mujeres directivas gira en torno al 27\%, lo que supone un claro estancamiento respecto a la tendencia al alza de los últimos diez años. Junto a la brecha de poder, también se ha de considerar la discriminación que la mujer experimenta como consecuencia de la división sexual del trabajo. En el caso de Europa, por ejemplo, esta sigue siendo relativamente alta, sobre todo en países como Estonia, Eslovaquia, Letonia y Finlandia (European Commission, 2009), donde las mujeres que trabajan en sectores como la construcción o la ingeniería representan una escasa minoría. En España esta segregación se ha visto aumentada en los últimos 15 años y, aunque no se encuentra entre los países con un mayor índice, llega a superar el promedio europeo. Del mismo modo, se constata que la incorporación de la mujer al mercado laboral no ha supuesto el logro de la corresponsabilidad en el ámbito familiar. Así, aunque el número de hogares sustentados por mujeres se haya incrementado en nuestro país 7 puntos porcentuales desde el año 2006, son ellas quienes se siguen haciendo cargo de las tareas domésticas y del cuidado de menores y personas ancianas y/o dependientes (Consejo Económico y Social, 2017).

Esta manifiesta discriminación se mantiene, en gran medida, sobre las creencias y actitudes estereotipadas que perviven arraigadas en la sociedad (European Institute of Gender Equality, 2017). Culturalmente aceptadas, estas ideas sobre los comportamientos, los roles y las características que la sociedad asocia a mujeres y a hombres, respectivamente, influyen sobre la conducta de los sujetos, llegando a convertirse en un importante obstáculo para el avance profesional femenino (Heilman, 2012). En algunas ocasiones, además, juegan un papel determinante en la contratación y vienen a reforzar la división sexual del trabajo (Koch, D'Mello y Sackett, 2015). La intensa resistencia al cambio que presentan provoca a su vez que, a pesar de la incorporación progresiva de la mujer al mundo profesional, su imagen en el orden simbólico no haya mejorado y apenas se perciban cambios en los últimos treinta años (Haines, Deaux y Lofaro, 2016).

En el contexto educativo, numerosas investigaciones han venido a señalar la existencia de estas representaciones culturales entre el alumnado de las diferentes etapas (Azorín, 2017; Ferragut, Blanca y Ortiz-Tallo, 2014; Ferragut, Blanca, Ortiz-Tallo y Bendayan, 2017; García Pérez et al., 2010; Miller, Eagly y Linn, 2015; Rodríguez, Lameiras, Carrera y Faílde, 2010). En el caso específico de la Formación Profesional, el estudio de Mosteiro y Porto (2017) dibuja un panorama similar, ya que aunque este alumnado parezca mostrar un posicionamiento igualitario, son las alumnas quienes se siguen oponiendo con mayor insistencia a la discriminación laboral de la mujer. Factores que pueden explicar esta tendencia es el escaso peso que el tratamiento transversal de la educación en valores tiene en el currículo de esta etapa (Ministerio de Educación, Cultura y Deporte, 2017a), Ilegando incluso a reforzarse las estructuras de género existentes en el ámbito laboral (Ledman, Rosvalll y Nylund, 2017). A ello, se ha de agregar también el acceso tradicionalmente sesgado que presentan este tipo de estudios, donde las estudiantes suelen ser mayoría en las familias profesionales que tradicionalmente han sido femeninas, como sanidad, imagen personal y servicios socioculturales y a la comunidad (Ministerio de Educación, Cultura y Deporte, 2017b). Ante esta situación, urgen actuaciones que permitan diagnosticar la cultura de género que posee el alumnado de Formación Profesional con el fin de mejorar las oportunidades laborales de mujeres y hombres y de la sociedad en general.

\section{OBJETIVO}

El presente trabajo tuvo como propósito reconocer las creencias estereotipadas que el alumnado de Formación Profesional puede presentar en el ámbito laboral, comprobando, además, si estas difieren según el sexo y el nivel de ciclo formativo cursado (grado medio y grado superior). 


\section{LA CULTURA DE GÉNERO DEL ALUMNADO DE FORMACIÓN PROFESIONAL EN EL ÁMBITO LABORAL}

\section{MUESTRA}

Para dar alcance a este objetivo, se contó con la participación voluntaria de 135 estudiantes de Formación Profesional de grado medio y superior que, en el momento de realizar la investigación, se encontraban cursando sus estudios en un centro integrado público de Formación Profesional de la provincia de Alicante. De estos, 76 eran hombres (56.30\%) y 59 mujeres $(43.70 \%)$, con edades comprendidas mayoritariamente entre los 16 y los 26 años (85.20\%). Respecto al nivel cursado, el $54.81 \%$ estaba matriculado en un ciclo formativo de grado medio y el $45.19 \%$ restante en uno de grado superior. Concretamente, tomó parte el alumnado del ciclo de Salud ambiental (22.22\%), Cuidados auxiliares de enfermería (20.74\%), Cocina y gastronomía (18.52\%), Instalaciones frigoríficas y climatización (17.78\%), Mantenimiento de instalaciones térmicas y fluidos (14.07\%) y Electromecánica de vehículos automóviles (6.67\%).

\section{METODOLOGÍA}

La medida empleada en el estudio fue la Escala de actitudes de rol de género (EARG) (GarcíaCueto et al., 2015), elaborada a partir de los instrumentos existentes en la literatura para la valoración del sexismo. Fundamentada en la teoría de la igualdad y la transformación de las creencias estereotipadas, permite identificar las actitudes de rol de género durante la etapa de la adolescencia y la juventud. Se trata de una escala tipo Likert con 5 puntos, cuyo formato de respuesta varía entre 1 ("totalmente en desacuerdo") y 5 ("totalmente de acuerdo"). Compuesta por 20 ítems, estos se estructuran en torno a dos categorías, actitudes trascendentes 0 igualitarias y actitudes sexistas. Mientras que la primera se subdivide en dos ámbitos, familiar y social, en la segunda se añade la esfera laboral a estas dos. La inclusión de los principales procesos implicados en la socialización del ser humano y el elevado índice de fiabilidad que presenta $(\alpha=.99)$ motivaron la selección de este instrumento para llevar a cabo la investigación. En concreto, en este trabajo se presentan los resultados referentes al Sexismo de función laboral.

Tras el logro de la autorización preceptiva por parte del equipo directivo, la recogida de los datos se realizó durante el horario lectivo habitual, en las aulas del centro y de manera grupal. La persona responsable de la aplicación de la prueba fue una de las investigadoras del equipo. Esta informó brevemente a los sujetos sobre los objetivos de la investigación y les facilitó las instrucciones para la cumplimentación de la escala. Para ello, se utilizaron tres sesiones con una duración aproximada de 20 minutos cada una de ellas. Durante estas se recordó en varias ocasiones el carácter anónimo de las respuestas, la confidencialidad de los datos y la voluntariedad en la participación. A pesar de ello, uno de los alumnos rehusó tomar parte en la investigación.

El tratamiento de los datos se realizó con el apoyo del software SPSS .20. Primeramente, se llevó a cabo un análisis descriptivo básico (media y desviación típica) y, con posterioridad, se aplicó la prueba $t$ de Student para verificar la existencia de diferencias estadísticamente significativas en los roles de género en el ámbito laboral, en función del sexo y del nivel de ciclo formativo cursado (grado medio y superior).

\section{RESULTADOS}

En un primer momento se presentan los resultados obtenidos en la muestra total y, seguidamente, aquellos hallados al comparar las actitudes de los sujetos según el sexo y el nivel de ciclo formativo en el que estos se encontraban matriculados (grado medio y superior).

\section{Roles de género de la muestra total en las actitudes sexistas en el ámbito laboral}

El análisis de los datos evidenció que las actitudes del alumnado respecto a la igualdad de género en el plano laboral no estaban claramente definidas. Más bien al contrario, estas parecían carac- 
terizarse por la ambigüedad y la incertidumbre. Tal y como se puede observar en la Tabla 1, el conjunto de la muestra no se manifestó a favor ni en contra al negar la existencia de profesiones eminentemente femeninas ni masculinas. Algo similar sucedió ante la exclusión de las mujeres de determinados puestos de trabajo y el rol del padre como sostén económico de la familia, afirmaciones ante las que los sujetos parecieron mostrar un cierto grado de indecisión. Una tendencia más igualitaria se apreció al aceptar la valoración social del trabajo de la pareja por encima del propio. Frente a ello, los sujetos rechazaron con más rotundidad la discriminación de la mujer en ocupaciones relevantes y en los cargos de responsabilidad.

Tabla 1

Roles de género en el ámbito laboral de la muestra total

\begin{tabular}{llll}
\hline Ítems & $M$ & $D E$ \\
\hline 1. & $\begin{array}{l}\text { Es preferible que los puestos de responsabilidad los ocupen los } \\
\text { hombres }\end{array}$ & 1.31 & .674 \\
2. La principal responsabilidad de un padre es ayudar & 2.24 & .125 \\
$\quad \begin{array}{l}\text { económicamente a sus hijos } \\
\text { 3. Algunos trabajos no son apropiados para las mujeres }\end{array}$ & 2.28 & 1.31 \\
4. Acepto que en mi círculo de amistades el trabajo futuro de mi & 2.01 & 1.31 \\
5areja se valore más que el mío & $\begin{array}{l}\text { Solo algunos tipos de trabajo son apropiados tanto para hombres } \\
\text { como para mujeres }\end{array}$ & 2.42 & 1.37 \\
6. $\begin{array}{l}\text { que muchos trabajos importantes es mejor contratar a hombres } \\
\text { ques }\end{array}$ & 1.68 & 1.13 \\
\hline
\end{tabular}

\section{Diferencias en las actitudes sexistas en la esfera laboral en función del sexo}

La aplicación de la prueba $t$ de Student puso de manifiesto la existencia de diferencias estadísticamente significativas en la mayoría de los ítems de la escala entre uno y otro sexo. Tal y como se recoge en la Tabla 2, fueron las alumnas quienes se mostraron más disconformes con la infrarrepresentación de las mujeres en los espacios de poder. Un planteamiento semejante se distinguió cuando volvieron a ser ellas quienes rechazaron más enérgicamente la contratación de los hombres en trabajos destacados. En ambos casos la magnitud de las diferencias con sus pares fue, además, moderada. Las participantes también rehusaron más firmemente que su trabajo fuera menos valorado socialmente que el de sus parejas. Ante esta afirmación, la magnitud de las diferencias fue, a su vez, notoriamente grande. Del mismo modo, fueron las estudiantes quienes se opusieron con mayor rotundidad a la división sexual del trabajo, mostrando ellos una postura más tendente hacia la ambigüedad y la indecisión. Tanto en uno como en otro caso la magnitud de las diferencias fue grande. Por el contrario, no se apreciaron diferencias estadísticamente significativas al valorar el papel del padre como fuente de ingresos para la economía familiar. 


\section{LA CULTURA DE GÉNERO DEL ALUMNADO DE FORMACIÓN PROFESIONAL EN EL ÁMBITO LABORAL}

Tabla 2

Diferencias en el Sexismo de función laboral según el sexo

\begin{tabular}{|c|c|c|c|c|c|c|c|c|}
\hline \multirow[t]{2}{*}{ Ítems } & \multicolumn{2}{|c|}{ Mujer } & \multicolumn{2}{|c|}{ Hombre } & \multicolumn{4}{|c|}{ Sig. Estadística } \\
\hline & $M$ & $D E$ & $M$ & $D E$ & $t$ & g.l. & $p$ & $d$ \\
\hline $\begin{array}{l}\text { 1. Es preferible que los puestos de } \\
\text { responsabilidad los ocupen los } \\
\text { hombres }\end{array}$ & 1.08 & .427 & 1.49 & .774 & 3.83 & 121 & $<.001$ & -.63 \\
\hline $\begin{array}{l}\text { 2. La principal responsabilidad del } \\
\text { padre es ayudar económicamente } \\
\text { a sus hijos }\end{array}$ & 2.02 & 1.22 & 2.41 & 1.25 & 1.81 & 133 & 0.72 & - \\
\hline $\begin{array}{l}\text { 3. Algunos trabajos no son } \\
\text { apropiados para las mujeres }\end{array}$ & 1.63 & 1.01 & 2.79 & 1.29 & 5.83 & 132 & $<.001$ & -.99 \\
\hline $\begin{array}{l}\text { 4. Acepto que en mi círculo de } \\
\text { amistades el trabajo futuro de mi } \\
\text { pareja se valore más que el mío }\end{array}$ & 1.29 & .720 & 2.57 & 1.40 & 6.84 & 116 & $<.001$ & -1.1 \\
\hline $\begin{array}{l}\text { 5. Solo algunos tipos de trabajo son } \\
\text { apropiados tanto para hombres } \\
\text { como para mujeres }\end{array}$ & 1.85 & 1.03 & 2.87 & 1.45 & 4.76 & 131 & $<.001$ & -.80 \\
\hline $\begin{array}{l}\text { 6. En muchos trabajos importantes } \\
\text { es mejor contratar a hombres que } \\
\text { a mujeres }\end{array}$ & 1.27 & .691 & 2.00 & 1.30 & 4.17 & 118 & $<.001$ & -.68 \\
\hline
\end{tabular}

\section{Diferencias en las actitudes sexistas en el terreno laboral en función del nivel de ciclo formativo}

En cuanto a la posible existencia de diferencias estadísticamente significativas, los resultados de la prueba $t$ de Student señalaron que estas se producían solo en algunos ítems de la escala (Tabla 3). En este contexto, fue el alumnado de los ciclos formativos de grado superior quien pareció expresar una posición más tendente hacia la igualdad en el ámbito laboral. Concretamente, se mostró más partidario de la presencia de la mujer en los cargos de responsabilidad y de la implicación del padre en la educación familiar. En el primer caso, el tamaño de las diferencias fue moderado, mientras que, en lo relativo al papel del progenitor como sostén económico de la familia, la magnitud del efecto fue algo mayor. En el resto de ítems no se apreciaron diferencias significativas estadísticamente.

Tabla 3

Diferencias en el Sexismo de función laboral según el nivel del ciclo formativo

\begin{tabular}{|c|c|c|c|c|c|c|c|c|c|}
\hline \multirow{2}{*}{\multicolumn{2}{|c|}{ Ítems }} & \multicolumn{2}{|c|}{ GM } & \multicolumn{2}{|c|}{ GS } & \multicolumn{4}{|c|}{ Sig. Estadística } \\
\hline & & \multirow{2}{*}{$\begin{array}{c}M \\
1.42\end{array}$} & \multirow{2}{*}{$\begin{array}{l}D E \\
.683\end{array}$} & \multirow{2}{*}{$\begin{array}{c}M \\
1.13\end{array}$} & \multirow{2}{*}{$\begin{array}{l}D E \\
.536\end{array}$} & \multirow{2}{*}{$\begin{array}{c}t \\
2.71\end{array}$} & \multirow{2}{*}{$\begin{array}{l}\text { g.l. } \\
131\end{array}$} & \multirow{2}{*}{$\begin{array}{c}p \\
.008\end{array}$} & \multirow{2}{*}{$\begin{array}{c}d \\
.47\end{array}$} \\
\hline 1. & $\begin{array}{l}\text { Es preferible que los puestos de } \\
\text { responsabilidad los ocupen los } \\
\text { hombres }\end{array}$ & & & & & & & & \\
\hline 2. & $\begin{array}{l}\text { La principal responsabilidad del } \\
\text { padre es ayudar económicamente } \\
\text { a sus hijos }\end{array}$ & 2.53 & 1.21 & 1.83 & 1.15 & 3.35 & 132 & .001 & .60 \\
\hline & $\begin{array}{l}\text { Algunos trabajos no son } \\
\text { apropiados para las mujeres }\end{array}$ & 2.23 & 1.29 & 2.30 & 1.30 & -.311 & 132 & .757 & - \\
\hline & $\begin{array}{l}\text { Acepto que en mi círculo de } \\
\text { amistades el trabajo futuro de mi } \\
\text { pareja se valore más que el mío }\end{array}$ & 2.07 & 1.24 & 1.95 & 1.41 & .511 & 132 & .610 & - \\
\hline & $\begin{array}{l}\text { Solo algunos tipos de trabajo son } \\
\text { apropiados tanto para hombres } \\
\text { como para mujeres }\end{array}$ & 2.57 & 1.39 & 2.27 & 1.35 & 1.25 & 132 & .210 & - \\
\hline & $\begin{array}{l}\text { En muchos trabajos importantes } \\
\text { es mejor contratar a hombres que } \\
\text { a mujeres }\end{array}$ & 1.74 & 1.11 & 1.55 & 1.09 & 1.00 & 132 & .316 & - \\
\hline
\end{tabular}




\section{DISCUSIÓN}

Esta investigación se planteó con el propósito de conocer las creencias estereotipadas que el alumnado de Formación Profesional poseía en el contexto laboral y comprobar, además, si estas variaban según el sexo y el nivel de ciclo formativo cursado (grado medio y superior). El análisis de los resultados lleva a indicar que los alumnos y las alumnas de esta etapa no se oponen con rotundidad a la discriminación de la mujer en el ámbito laboral, especialmente en lo concerniente a la organización sexual del trabajo y al modelo del padre como sustentador de la familia. Estos resultados son concomitantes con los hallados por Mosteiro y Porto (2017), quienes destacaron que a pesar de mostrar actitudes aparentemente igualitarias, el alumnado de Formación Profesional sigue manteniendo una cultura de género claramente estereotipada y sexista. Hallazgos similares se advierten también en otros niveles educativos, como Educación Primaria (Azorín, 2017), Educación Secundaria (Rodríguez et al., 2010) e incluso en la educación superior, donde el estudio transnacional de Miller et al. (2015) señaló que el alumnado universitario consideraba que las mujeres estaban menos capacitadas que sus pares para las profesiones científico-tecnológicas.

El análisis de las diferencias en función del sexo puso de manifiesto que eran ellas quienes se mostraban más contrarias ante el sexismo laboral, sobre todo en lo referente a la discriminación de la mujer en los espacios de poder y responsabilidad y a la infravaloración de su trabajo. Este menor nivel de estereotipos de género femeninos también ha sido recogido en otras investigaciones, que vienen a señalar que siguen siendo los jóvenes quienes manifiestan un grado más elevado de creencias sexistas (Ferragut et al., 2014; García Pérez et al., 2010). En cuanto a las posibles divergencias existentes según el nivel de ciclo formativo cursado, los resultados sugirieron que, en líneas generales, las creencias estereotipadas no presentaban una importante variación. Solo se apreciaron diferencias significativas a la hora de rechazar la segregación vertical y el rol del padre como sostén económico de la familia, siendo en ambos casos a favor del alumnado de grado superior. Mosteiro y Porto (2017) también obtuvieron resultados equivalentes, señalando que son los alumnos y las alumnas de los niveles más avanzados quienes parecen poseer actitudes menos sexistas. Esta variabilidad de las creencias estereotipadas con la edad ha sido subrayada también por Ferragut et al. (2017), quienes evidenciaron que, a diferencia del sexismo hostil, el benevolente disminuye durante la adolescencia.

Ante tales hallazgos, se considera necesaria la puesta en marcha de actuaciones que permitan el reconocimiento y transformación de este tipo de creencias entre el alumnado de Formación Profesional. Los centros educativos no pueden permanecer ajenos a los desafíos que plantea la sociedad actual y, especialmente, a los obstáculos y barreras que la mujer ha de superar en el contexto laboral. Aunque se hayan conseguido metas esenciales en este ámbito y la mujer haya podido salir del espacio doméstico, el logro de un mercado de trabajo justo y equitativo con todos sus empleados y empleadas exige aún la superación de importantes retos. Para ello, se ha de partir del compromiso social y moral de todas las instituciones encargadas de la formación humana y axiológica del alumnado, lo que implica, inexorablemente, la responsabilidad de los centros educativos y de los agentes que los integran. Solo mediante la intervención generalizada, continua y eminentemente curricular en esta área se logrará aprovechar el potencial de todo el capital humano de la sociedad.

\section{REFERENCIAS}

Azorín, C. M. (2017). Actitudes hacia la igualdad de género en una muestra de estudiantes de Murcia. Revista Complutense de Educación, 28(1), 45-60. DOI: 10.5209/rev_RCED.2017.v28.n1.48715 


\section{LA CULTURA DE GÉNERO DEL ALUMNADO DE FORMACIÓN PROFESIONAL EN EL ÁMBITO LABORAL}

Burton, L. J. (2015). Underrepresentation of women in sport leadership: a review of research. Sport Management Review, 18, 155-165. D0I: 10.1016/j.smr.2014.02.004

Catalyst. (2017). Women CEOS of the S\&P 500. Recuperado de: http://www.catalyst.org

Consejo Económico y Social. (2017). Informe 05/2016. La participación laboral de las mujeres en España. Recuperado de: http://www.ces.es/documents/10180/3557409/Inf0516.pdf

European Commission. (2009). Gender segregation in the labour market Root causes, implications and policy responses in the EU. Recuperado de: http://ec.europa.eu/social/BlobServlet?docld=4028

European Institute of Gender Equality. (2017). Gender segregation in education, training and the labour market. Recuperado de: http://eige.europa.eu/about-eige/procurement/eige-2016-oper13

European Union. (2017). 2017 Report on equality between women and men in the EU. Belgium: European Union.

Ferragut, M., Blanca, M. J. y Ortiz-Tallo, M. (2014). Analysis of adolescent profiles by gender: strengths, attitudes toward violence and sexism. Spanish Journal of Psychology, 17(59), 1-10. DOI: 10.1017/sjp.2014.60.

Ferragut, M., Blanca, M. J., Ortiz-Tallo, M. y Bendayan, R. (2017). Sexist attitudes and beliefs during adolescence: a longitudinal study of gender differences. European Journal of Developmental Psychology, 14(1), 32-43. DOI: 10.1080/17405629.2016.1144508

Gagliarducci, S. y Paserman, M. D. (2012). Gender interactions within hierarchies: evidence from the political arena. Review of Economic Studies, 79, 1021-1052. D0I: 10.1093/restud/rdr046

García Pérez, R., Rebollo, M. A., Buzón, 0., González-Piñal, R., Barragán, R. y Ruiz, E. (2010). Actitudes del alumnado hacia la igualdad de género. Revista de Investigación Educativa, 28(1), 217-232.

García-Cueto, E., Rodríguez-Díaz, F. J., Bringas-Molleda, C., López-Cepero, J., Paino-Quesada, S. y Rodríguez-Franco, L. (2015). Development of the Gender Role Attitudes Scale (GRAS) amongst young Spanish people. International Journal of Clinical and Health Psychology, 15(1), 61-68. DOI: 10.1016/j.ijchp.2014.10.004

Govender, V. y Penn-Kekana, L. (2008). Gender biases and discrimination: a review of health care interpersonal interactions. Global Public Health, 3(S1), 90-103. D0I: 10.1080/17441690801892208.

Grant Thornton. (2017). Women in business. New perspectives on risk and reward. Recuperado de: http://www.grantthornton.co.uk/globalassets/1.-memberfirms/united-kingdom/pdf/publication/women-in-business-new-perspectives-onrisk-and-reward.pdf

Haines, E. L., Deaux, K. y Lofaro, N. (2016). The times they are a-changing ... or are they not? A comparison of gender stereotypes, 1983-2014. Psychology of Women Quarterly, 40(3), 353363. DOI: $10.1177 / 0361684316634081$

Heilman, M. E. (2012). Gender stereotypes and workplace bias. Research in Organizational Behavior, 32, 113-135. DOl: 10.1016/j.riob.2012.11.003

INE. (2017). Mujeres y hombres en España. Recuperado de: http://www.ine.es

Koch, A. J., D'Mello, S. D. y Sackett, P. R. (2015). A meta-analysis of gender stereotypes and bias in experimental simulations of employment decision making. Journal of Applied Psychology, 100, 128-161. DOI: 10.1037/a0036734

Ledman, K., Rosvall, P. y Nylund, M. (2017). Gendered distribution of "knowledge required for empowerment" in Swedish vocational education curricula? Journal of Vocational Education \& Training, 70(1), 85-106. DOI: 10.1080/13636820.2017.1394358 
Miller, D. I., Eagly, A. H. y Linn, M. C. (2015). Women's representation in science predicts national gender-science stereotypes: evidence from 66 nations. Journal of Educational Psychology, 107(3), 631-644. DOI: 10.1037/edu0000005

Ministerio de Educación, Cultura y Deporte (2017a). Currículos del Ministerio de Educación. Recuperado de: http://todofp.es/que-como-y-donde-estudiar/que-estudiar/nuevos-titulos/curriculos-ministerio-educacion.html

Ministerio de Educación, Cultura y Deporte (2017b). Enseñanzas no universitarias. Alumnado matriculado. Curso 2015-2016. Resultados detallados. Recuperado de: http://www.educacion.es/servicios-al-ciudadano-mecd/ca/estadisticas/educacion/no-universitaria/alumnado/matriculado/2015-2016-RD.html

Morrison, Z., Bourke, M. y Kelley, C. (2005). "Stop making it such a big issue": perceptions and experiences of gender equality by undergraduates at a British university. Women's Studies International Forum, 28, 150-162.

Mosteiro, M. J. y Porto, A. M. (2017). Análisis de los estereotipos de género en alumnado de Formación Profesional: diferencias según sexo, edad y grado. Revista de Investigación Educativa, 35(1), 151-165.

Rodríguez, Y., Lameiras, M., Carrera, M. V. y Faílde, J. M. (2010). Evaluación de las actitudes sexistas en estudiantes españoles/as de Educación Secundaria Obligatoria. Psychologia: Avances de la disciplina, 4(1), 11-24.

U. S. Bureau of Labor Statistics. (2015). BLS Reports. Women in the labor force: a databook. Recuperado de: https://www.bls.gov/opub/reports/womensdatabook/archive/women-in-thelabor-force-a-databook-2015.pdf 
